\title{
Recent developments in modeling of solid-state fermentation: heat and mass transfer in bioreactors
}

\author{
David A. Mitchell ${ }^{\mathrm{a}, *}$, Oscar F. von Meien ${ }^{\mathrm{b}}$, Nadia Krieger ${ }^{\mathrm{c}}$ \\ a Departamento de Bioquímica e Biologia Molecular, Universidade Federal do Paraná, Cx.P. 19046, Jardim das Américas, \\ Curitiba 81531-990, Paraná, Brazil

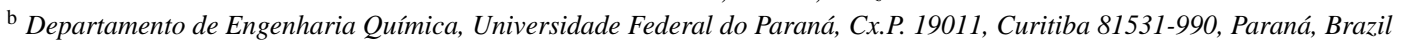 \\ c Departamento de Química, Universidade Federal do Paraná, Cx.P. 19081, 81531-990 Curitiba, Paraná, Brazil
}

Received 7 December 2001; accepted after revision 24 July 2002

\begin{abstract}
Mathematical models are important tools for optimizing the design and operation of solid-state fermentation (SSF) bioreactors. Such models must describe the transport phenomena within the substrate bed and mass and energy exchanges between the bed and the other subsystems of the bioreactor, such as the bioreactor wall and headspace gases. The sophistication with which this has been done for SSF has improved markedly over the last decade or so. The current article reviews these advances, showing how the various transport phenomena have been modeled. It also discusses the insights that have been achieved through the modeling work and the improvements to models that will be necessary in order to make them even more powerful tools in the optimization of bioreactor performance.
\end{abstract}

(C) 2002 Elsevier Science B.V. All rights reserved.

Keywords: Solid-state fermentation; Bioreactors; Modeling; Packed-bed bioreactors; Rotating-drum bioreactors; Gas-solid fluidized bed bioreactors; Tray bioreactors

\section{Introduction}

Mathematical modeling is an essential tool for optimizing bioprocesses. Not only can models guide the design and operation of bioreactors but they can also provide insights into how the various phenomena within the fermentation system combine to control overall process performance. However, it is only over the last decade or so that concerted efforts have been made to develop mathematical models of solid-state fermentation (SSF) processes. The present work reviews the advances that have been made over this period.

The various mathematical models that have been proposed to date in the area of SSF can be divided into two categories, macroscale models and microscale models [1]. Macroscale models are those that concern themselves with the operation of bioreactors. They describe mass and heat transport processes across the substrate bed, but typically treat phenomena at the level of the individual particle in a highly simplified manner. As such, they could also be referred to as SSF bioreactor models. Microscale models are those that concern themselves with the phenomena that occur on and

\footnotetext{
* Corresponding author. Fax: +55-41-2660-222.

E-mail address: davimitc@engquim.ufpr.br (D.A. Mitchell).
}

within individual particles and do not attempt to describe the performance of the bioreactor as a whole. Clearly, the performance of an SSF bioreactor can potentially be limited either by the phenomena that occur at the level of the whole bed or by the phenomena that occur within individual particles, so both types of models are important, despite their differing objectives.

The aim of SSF bioreactor models is to describe how the performance is affected by the various operating variables that can be manipulated in an attempt to control the process. For example, a mathematical model will enable predictions of how the flow rate, humidity and temperature of the inlet air will affect the temperature and water content of the substrate bed, and in turn, how these system variables will affect microbial growth and product formation. Therefore bioreactor models can be thought of as consisting of two sub-models: the kinetic sub-model and the balance/transport sub-model (Fig. 1). The balance/transport sub-model describes mass and heat transfer within and between the various phases of the bioreactor, while the kinetic sub-model describes how the growth rate of the microorganism depends on the key local environmental variables.

Mathematical modeling in SSF is reviewed in two parts. The current part reviews the balance/transport sub-models 


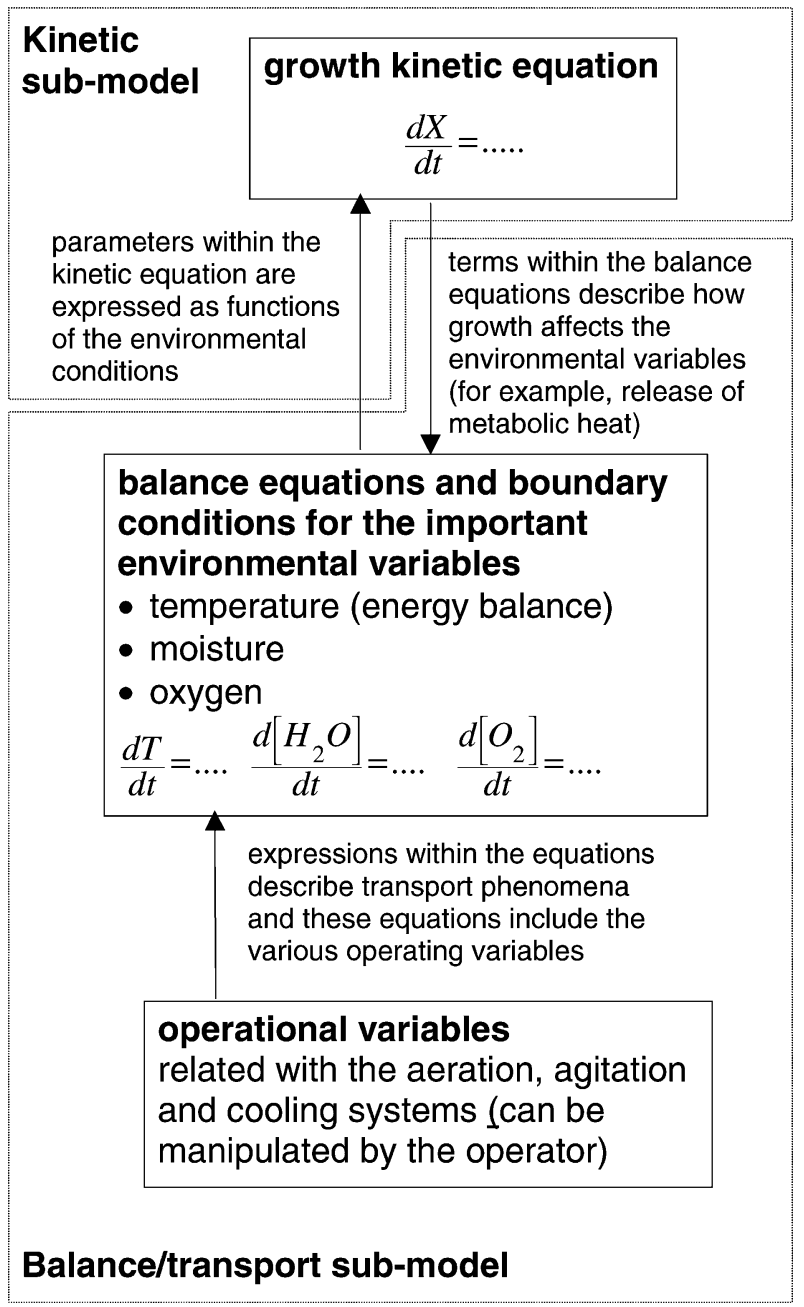

Fig. 1. The structure of bioreactor models, showing how they are composed of kinetic and balance/transport sub-models.

of bioreactors. Part II reviews both the kinetic sub-models of bioreactor models and microscale models of those processes associated with growth that occur at the level of individual particles [2]. Both these reviews aim to give an insight into how the various phenomena that occur within the system can be described mathematically. To this end key model equations are reproduced and discussed. However, the sets of equations that constitute the various models that are discussed are not reproduced in their entirety. Readers wishing to understand individual models in detail must refer to the original works. Also discussed are the insights that have been achieved through the modeling work, and improvements to models that will be necessary in the future. The current part of the review is organized on the basis of the various bioreactor types that are used in SSF. In presenting the balance equations, the terms linked with growth, for example heat production and $\mathrm{O}_{2}$ consumption, are replaced with a simple rate term, since the mathematical form of such terms is the subject of Part II [2].

\section{State of the art in modeling mass and energy balances in tray bioreactors}

In the first models of tray bioreactors, pseudo-steady-state approximations were made in order to investigate specific aspects of tray bioreactor performance. Ragheva Rao et al. [3] made a pseudo-steady-state approximation for $\mathrm{O}_{2}$ diffusion with the bed to arrive at an algebraic equation that gave the $\mathrm{O}_{2}$ concentration profile as a function of height within the bed. This was used to calculate a critical bed height. At bed heights greater than the critical height, $\mathrm{O}_{2}$ limitation occurred in the deeper regions of the bed during the fermentation. However, as later work showed that high temperatures are a more important limiting factor in tray bioreactors than $\mathrm{O}_{2}$ exhaustion, this concept is of limited usefulness. Szewczyk [4] wrote a pseudo-steady-state energy balance to arrive at an algebraic equation that gave the temperature profile in the bed as a function of depth and used this equation to explore the effectiveness of surface cooling in controlling the temperature within the bed.

The more recent models of Rajagopalan and Modak [5,6] and Smits et al. [7] consider both mass and energy balances and do not make the pseudo-steady-state assumption. In these two models, the systems are visualized slightly differently (Fig. 2).

\subsection{Oxygen balances for tray bioreactors}

The balance equations for $\mathrm{O}_{2}$ within the bed have terms for $\mathrm{O}_{2}$ diffusion within the pores and uptake by the microorganism. However, due to the different ways in which the system was visualized, the equations appear slightly differently. The equation used by Smits et al. [7] to describe the $\mathrm{O}_{2}$ balance within the bed is relatively simple:

$\frac{\partial C_{\mathrm{O}_{2}}^{\mathrm{b}}}{\partial t}=D_{\mathrm{O}_{2}}^{\mathrm{b}} \frac{\partial^{2} C_{\mathrm{O}_{2}}^{\mathrm{b}}}{\partial z^{2}}-r_{\mathrm{O}_{2}}$

where $t$ is the time, $C_{\mathrm{O}_{2}}^{\mathrm{b}}$ the concentration of $\mathrm{O}_{2}$ per unit volume of the bed, $z$ the vertical coordinate and $r_{\mathrm{O}_{2}}$ is rate of $\mathrm{O}_{2}$ uptake by the microorganism. The first term on the right-hand side (RHS) describes the diffusion of $\mathrm{O}_{2}$ within the pores, the diffusivity $D_{\mathrm{O}_{2}}^{\mathrm{b}}$ being an effective diffusivity that accounts for the fact that the pores represent only part of the overall volume of the bed and provide a tortuous path for diffusion.

The model of Rajagopalan and Modak [5] recognizes the existence of a biofilm of biomass covering the particles within the bed. In this case, the equation for $\mathrm{O}_{2}$ transfer within the pores of the bed is

$\frac{\partial C_{\mathrm{O}_{2}} \varepsilon}{\partial t}=D_{\mathrm{O}_{2}}^{\mathrm{b}} \frac{\partial^{2} C_{\mathrm{O}_{2}}}{\partial z^{2}}-K_{\mathrm{a}} a_{\mathrm{x}}\left(C_{\mathrm{O}_{2}}-H C_{\mathrm{O}_{2}}^{\mathrm{f}}\right)$

where $C_{\mathrm{O}_{2}}$ is the concentration of $\mathrm{O}_{2}$ within the pores, $\varepsilon$ the porosity of the bed, $K_{\mathrm{a}}$ the mass transfer coefficient for $\mathrm{O}_{2}$ at the air/biofilm interface, $a_{\mathrm{x}}$ the area of the air/biofilm 

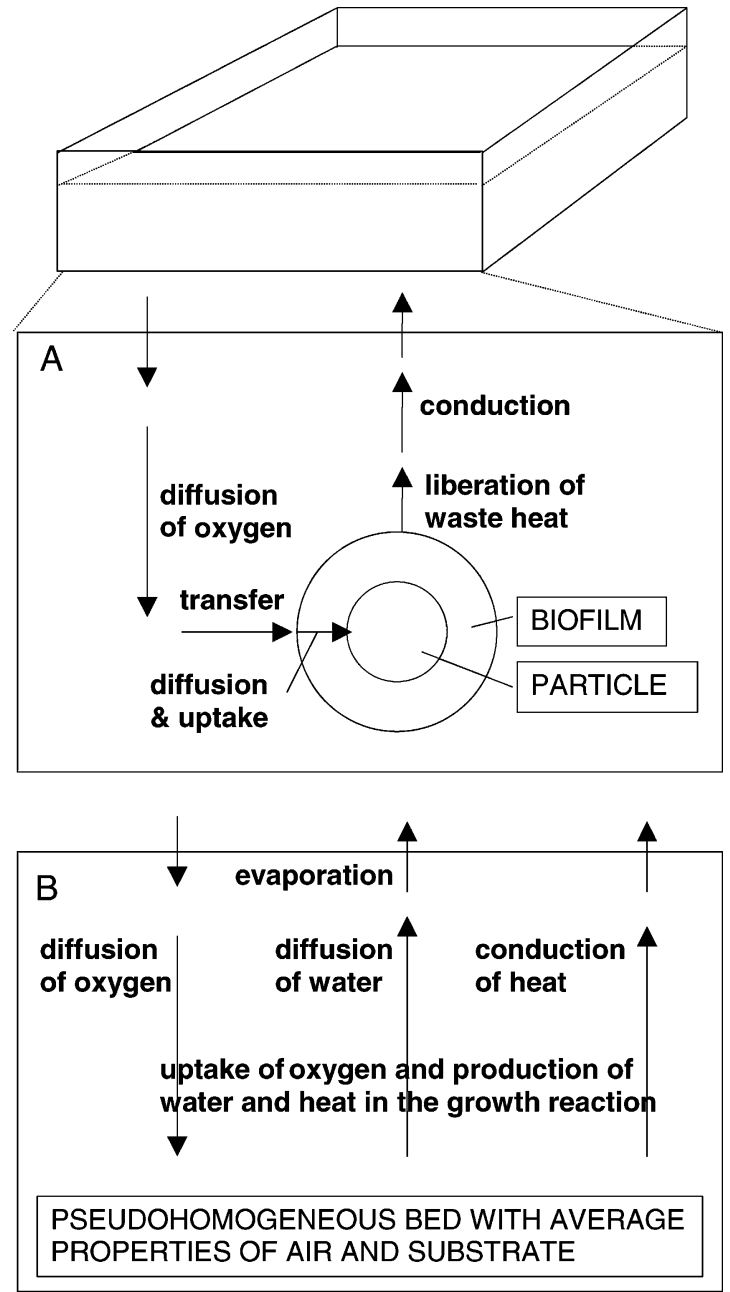

Fig. 2. Comparison of the manners in which the tray models of (A) Rajagopalan and Modak [5,6] and (B) Smits et al. [7] treat the tray system.

interface per unit volume of the bioreactor, $H$ the Henry's law constant and $C_{\mathrm{O}_{2}}^{\mathrm{f}}$ the concentration of $\mathrm{O}_{2}$ within the biofilm. Another equation is required to describe $\mathrm{O}_{2}$ uptake by the biomass. A pseudo-steady-state approximation was used to describe the rate of transfer of $\mathrm{O}_{2}$ into the biofilm:

$\frac{\partial C_{\mathrm{O}_{2}}^{\mathrm{f}}}{\partial t}=0=K_{\mathrm{a}} a_{\mathrm{x}}\left(C_{\mathrm{O}_{2}}-H C_{\mathrm{O}_{2}}^{\mathrm{f}}\right)-r_{\mathrm{O}_{2}}$

This implies that the film $\mathrm{O}_{2}$ concentration $\left(C_{\mathrm{O}_{2}}^{\mathrm{f}}\right)$ is assumed to adjust such that the rate of $\mathrm{O}_{2}$ transfer is equal to the rate of $\mathrm{O}_{2}$ uptake, which was assumed to depend on $\mathrm{O}_{2}$ concentration according to Monod kinetics.

In this model, the change in gas concentration as it flows in the headspace across the bed surface was also modeled. It is described by

$\frac{\partial C_{\mathrm{O}_{2}}^{\mathrm{x}}}{\partial t}=-v_{\mathrm{x}} \frac{\partial C_{\mathrm{O}_{2}}^{\mathrm{x}}}{\partial x}-\left.\frac{D_{\mathrm{O}_{2}}^{\mathrm{b}}}{z_{\mathrm{h}}} \frac{\partial C_{\mathrm{O}_{2}}}{\partial z}\right|_{y=L}$

where $C_{\mathrm{O}_{2}}^{\mathrm{x}}$ is the concentration of $\mathrm{O}_{2}$ in the headspace, $x$ the horizontal coordinate in the headspace, $L$ the height of the bed and $z_{\mathrm{h}}$ the height of the headspace. The first term on the RHS describes convection in the headspace across the surface of the bed and the second term describes transfer from the headspace to the bed.

In their later version of this model [6], the porosity was not a constant but a function of growth and the biofilm was not treated as though it were well mixed, such that the $\mathrm{O}_{2}$ concentration at the surface of the biofilm $\left(\left.C_{\mathrm{O}_{2}}^{\mathrm{f}}\right|_{R(t)}\right)$, appears explicitly in the term that describes transfer of $\mathrm{O}_{2}$ across the gas/biofilm interface:

$\frac{\partial C_{\mathrm{O}_{2}} \varepsilon(t)}{\partial t}=D_{\mathrm{O}_{2}}^{\mathrm{b}} \frac{\partial^{2} C_{\mathrm{O}_{2}}}{\partial z^{2}}-K_{\mathrm{a}} a_{\mathrm{x}}\left(C_{\mathrm{O}_{2}}-\left.H C_{\mathrm{O}_{2}}^{\mathrm{f}}\right|_{R(t)}\right)$

and another partial differential equation was used to describe the $\mathrm{O}_{2}$ diffusion and reaction within the biofilm itself:

$\frac{\partial C_{\mathrm{O}_{2}}^{\mathrm{f}}}{\partial t}=\frac{D_{\mathrm{O}_{2}}^{\mathrm{f}}}{r^{2}} \frac{\partial}{\partial r}\left(r^{2} \frac{\partial C_{\mathrm{O}_{2}}^{\mathrm{f}}}{\partial r}\right)-r_{\mathrm{O}_{2}}$

where $r$ is the radial coordinate within the substrate particle. The first term on the RHS describes diffusion of $\mathrm{O}_{2}$ within a sphere. The radius of this sphere increases during growth due to expansion of the biofilm.

Which of these approaches is better for the modeling of $\mathrm{O}_{2}$ transfer within bioreactors depends on the aim of the modeling and the experimental information available. The necessity to write an $\mathrm{O}_{2}$ balance for the headspace as was done by Rajagopalan and Modak [5] is unclear since experimental attention has not been given to headspace $\mathrm{O}_{2}$ concentrations. The later model of Rajagopalan and Modak [6] explicitly describes the various steps in $\mathrm{O}_{2}$ transfer to and within a biofilm growing at the particle surface and therefore is more mechanistic, providing the basis for an investigation into whether diffusion of $\mathrm{O}_{2}$ within the pores, transfer from the pores to the biofilm or diffusion within the biofilm is the limiting step in $\mathrm{O}_{2}$ transfer. However, if there is good empirical data on which to express $\mathrm{O}_{2}$ consumption rates, then the approach of Smits et al. [7] might be preferable due to its simplicity.

\subsection{Water balances for tray bioreactors}

To date only the model of Smits et al. [7] describes the water balance in the substrate bed within the tray. It is described by the following equation:

$\frac{\partial C_{\mathrm{w}}}{\partial t}=r_{\mathrm{H}_{2} \mathrm{O}}-\left[\frac{\partial C_{\mathrm{VAP}}}{\partial t}-D_{\mathrm{VAP}}^{*} \frac{\partial^{2} C_{\mathrm{VAP}}}{\partial z^{2}}\right]$

where $C_{\mathrm{W}}$ is the liquid water concentration per unit volume of bed, $C_{\mathrm{VAP}}$ the water vapor concentration per unit volume of bed, $D_{\mathrm{VAP}}^{*}$ the effective diffusion coefficient of water vapor within the bed and $r_{\mathrm{H}_{2} \mathrm{O}}$ the metabolic rate of water production. The first term within the square brackets on the RHS represents the evaporation of water. The rate of evaporation depends on the rate of change in the bed temperature, given that the air is assumed to be in equilibrium with the 
solid. The second term within these brackets represents the diffusion of water vapor within the void spaces.

However, the necessity for a water balance is not clear. Simulations with the model suggested that the diffusion of water was not important for a tray incubated in a $98 \%$ relative humidity headspace [7]. It might become important if the tray were incubated within a drier atmosphere in order to promote evaporative cooling, although such a strategy would quickly lead to drying out of the bed surface.

\subsection{Energy balances for tray bioreactors}

The energy balance in the model of Rajagopalan and Modak [5,6] takes into account conduction (the first term on the RHS) and metabolic heat production (the second term on the RHS):

$\rho_{\mathrm{s}} C_{p \mathrm{~s}} \frac{\partial T}{\partial t}=k_{\mathrm{b}} \frac{\partial^{2} T}{\partial z^{2}}+r_{Q}$

where $\rho_{\mathrm{s}}$ is the density of the bed, $C_{p \mathrm{~s}}$ the heat capacity of the bed, $T$ the bed temperature, $k_{\mathrm{b}}$ the thermal conductivity of the bed and $r_{Q}$ the rate of metabolic heat production by the microorganism. The balance of Smits et al. [7] has an additional term to describe evaporative heat removal (the last term on the RHS):

$\frac{\partial H}{\partial t}=k_{\mathrm{b}} \frac{\partial^{2} T}{\partial z^{2}}+r_{Q}+\lambda D_{\mathrm{VAP}}^{*} \frac{\partial^{2} C_{\mathrm{VAP}}}{\partial z^{2}}$

where $H$ is the enthalpy of the bed, which is a function of temperature and $\lambda$ the enthalpy of vaporization of water. The second term on the RHS describes conduction within the bed, whereas the third term on the RHS describes the removal of heat by the evaporation and diffusion of water, with the airspace in the pores of the bed assumed to be in moisture and thermal equilibrium with the solid. However, in typical use of tray bioreactors the evaporation term is unlikely to be necessary, since the simulations of Smits et al. [7] showed that the contribution of evaporation to cooling would be negligible if the tray were incubated in a $98 \%$ relative humidity environment.

\subsection{Key insights obtained from tray bioreactor models and future improvements needed}

Rajagopalan and Modak [5] used their model to explore the interactions between bed height and headspace air temperature, concluding that for adequate temperature control the optimum strategy was to use a thin bed, of around $1-3 \mathrm{~cm}$ height, and a headspace air temperature near the optimum temperature for growth.

Oxygen limitation within the biofilm is unavoidable, even when the $\mathrm{O}_{2}$ concentration in the gas phase at the surface of the biofilm is high [6]. Of course, this limitation is intrinsic to all SSF processes, regardless of bioreactor type [8]. In trays that allow for $\mathrm{O}_{2}$ exchange with the headspace only at the upper surface of the bed, $\mathrm{O}_{2}$ supply to the biofilm can become limiting in the lower regions of the bed, although the importance of this effect in relation to heat transfer limitations depends on the height of the bed and the relative values of the effective $\mathrm{O}_{2}$ diffusivity and the thermal conductivity [7].

The limitation of mass and heat transfer in trays to diffusion and conduction limits the ability to influence the environmental conditions within the bed of a tray. As such, further mathematical modeling work will probably be of limited use in the further optimization of design and operation of individual trays. However, the question of air flow within the headspace of the chamber does deserve some attention. Further, from the point of view of understanding trays better, modeling and experimental work could be done to evaluate the importance of natural convection within the bed, which can potentially occur due to the temperature gradients that arise.

\section{State of the art in the modeling of packed-bed bioreactors}

Although packed-bed bioreactors do not necessarily have to be vertical, in this section it is assumed that the bioreactor sits in a vertical orientation and is aerated from the bottom, as shown in Fig. 3, and the term horizontal conduction is used to denote conduction normal to the direction of the air flow. The symbol $z$ is used to denote vertical position, the symbol $x$ to denote horizontal position in rectangular geometry and the symbol $r$ to denote horizontal position in cylindrical geometry. Note that it is not necessary to model $\mathrm{O}_{2}$ uptake in packed-bed bioreactors because the forced aeration means that $\mathrm{O}_{2}$ will not be limiting. In fact, aeration rates are determined by the need to remove heat rather than the need to supply $\mathrm{O}_{2}$.

\subsection{Energy balances within packed-bed bioreactors}

The first models of packed-bed bioreactors did not include the contribution of evaporation to the energy balance and even some more recent models omit evaporation [9-11]. The energy balance of Sangsurasak and Mitchell [12] does include evaporation and describes heat transfer in both the vertical and radial directions within a cylindrical geometry:

$$
\begin{aligned}
\rho_{\mathrm{b}} & C_{p \mathrm{~b}}\left(\frac{\partial T}{\partial t}\right)+\rho_{\mathrm{a}}\left(C_{p \mathrm{a}}+f \lambda\right) V_{z}\left(\frac{\partial T}{\partial z}\right) \\
= & {\left[\frac{k_{\mathrm{b}}}{r}\left(\frac{\partial T}{\partial r}\right)+k_{\mathrm{b}}\left(\frac{\partial^{2} T}{\partial r^{2}}\right)\right]+k_{\mathrm{b}}\left(\frac{\partial^{2} T}{\partial z^{2}}\right)+r_{Q} }
\end{aligned}
$$

where $C_{p \mathrm{~b}}$ is the heat capacity of the bed, $\rho_{\mathrm{b}}$ the density of the bed, $C_{p \mathrm{a}}$ the heat capacity of the moist air, $\rho_{\mathrm{a}}$ the density of the air and $V_{z}$ the superficial velocity of the air. On the RHS, the term within the square brackets describes radial conduction while the second term describes axial conduction. The term that deserves attention is the second term 


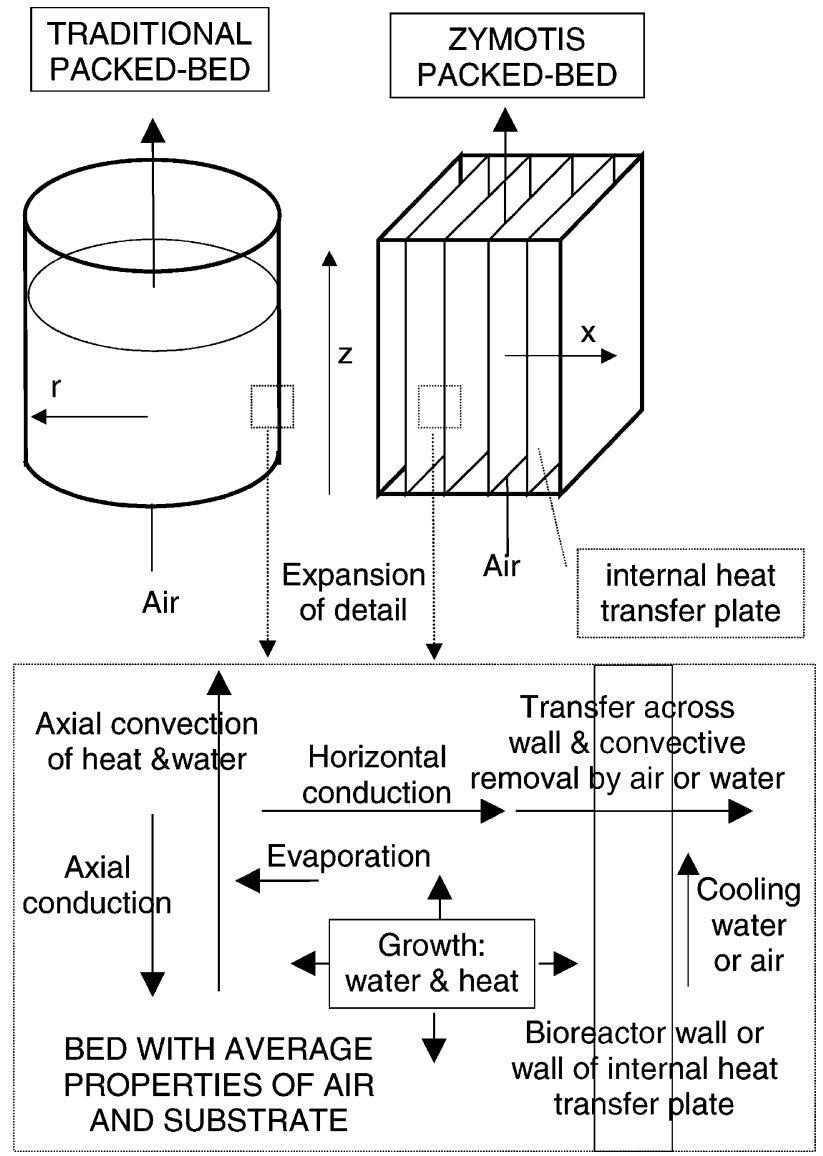

Fig. 3. Traditional and Zymotis packed-bed bioreactors and the various heat and mass transfer processes that must be modeled. Note that for wide traditional packed-bed bioreactors the removal of heat through the side wall may be negligible.

on the left-hand side (LHS), which describes both convective and evaporative heat removal. The combination $f \lambda$ arises from the assumption that the air remains saturated as it flows through the bed: the evaporation of water to maintain saturation gives the air a higher apparent heat capacity. Ideally the saturation water content of the air should be described by the Antoine equation but the linear approximation that is used in this equation is reasonable over temperature ranges of about $20^{\circ} \mathrm{C}$.

This equation has also been used to describe the situation where the bed is wide enough for horizontal conduction to make a negligible contribution to heat removal, in which case it is simply necessary to remove the terms within the square brackets on the RHS of Eq. (10) to give the following equation $[13,14]$ :

$$
\begin{aligned}
& \rho C_{p \mathrm{~b}}\left(\frac{\partial T}{\partial t}\right)+\left(\rho_{\mathrm{a}} C_{p \mathrm{a}}+\rho_{\mathrm{a}} f \lambda\right) V_{z}\left(\frac{\partial T}{\partial z}\right) \\
& =k_{\mathrm{b}}\left(\frac{\partial^{2} T}{\partial z^{2}}\right)+r_{Q}
\end{aligned}
$$

To describe the energy balance within the Zymotis bioreactor, the axial conduction term can be removed because it is small compared to axial convection and replaced by a term describing horizontal conduction to the heat transfer plates within the bed [15]:

$$
\begin{aligned}
\rho_{\mathrm{b}} & C_{p \mathrm{~b}}\left(\frac{\partial T}{\partial t}\right)+\rho_{\mathrm{a}}\left(C_{p \mathrm{a}}+f \lambda\right) V_{z}\left(\frac{\partial T}{\partial z}\right) \\
= & k_{\mathrm{b}}\left(\frac{\partial^{2} T}{\partial x^{2}}\right)+r_{Q}
\end{aligned}
$$

Only one horizontal direction is described since is assumed that horizontal heat transfer parallel to the heat transfer plates is negligible.

\subsection{Combined mass and energy balances within packed-bed bioreactors}

In the modeling studies cited above, although evaporation was taken into account in the energy balance, no water balance was included in the model. These models therefore allow all the water in the substrate to evaporate without predicting a decrease in growth rate. Such models are appropriate only for situations in which the evaporation of water during the fermentation does not significantly decrease the water activity of the substrate. However, this is often not the case and therefore Weber et al. [16] wrote both energy and water balances, using the pseudo-steady-state approximation. The energy balance is

$$
0=r_{Q}+F_{\text {air }} \frac{\begin{array}{l}
\mathrm{d}\left(C_{p \mathrm{~g}}\left(T-T_{\mathrm{ref}}\right)\right. \\
\left.+y_{\mathrm{VAP}}\left(C_{p \mathrm{VAP}}\left(T-T_{\mathrm{ref}}\right)+\lambda\right)\right)
\end{array}}{\mathrm{d} z}
$$

where $F_{\text {air }}$ is the air mass flow rate, $T_{\text {ref }}$ the reference temperature for the enthalpy values, $y_{\mathrm{VAP}}$ the gas phase humidity, $C_{p \mathrm{~g}}$ the heat capacity of dry air and $C_{p \mathrm{VAP}}$ the heat capacity of water vapor. In this equation, $y_{\mathrm{VAP}}$ is derived from the Antoine equation.

The balance for extracellular water is

$$
\begin{aligned}
(1-\varepsilon) C_{\mathrm{s}} \frac{\mathrm{d} X_{\mathrm{ws}}}{\mathrm{d} t}= & r_{\mathrm{H}_{2} \mathrm{Oext}}-(1-\varepsilon) X_{\mathrm{ws}} \frac{\mathrm{d} C_{\mathrm{s}}}{\mathrm{d} t} \\
& -F_{\text {air }} \frac{y_{\text {out }}-y_{\text {in }}}{H}
\end{aligned}
$$

where $C_{\mathrm{s}}$ is the mass of dry solids per unit volume of the bioreactor, $X_{\mathrm{ws}}$ the mass ratio of water to dry solids, $r_{\mathrm{H}_{2} \mathrm{O} \text { ext }}$ the rate of generation of extracellular water by the growth reaction and $H$ the height of the bed. The second term on the RHS describes the change in extracellular water content due to the consumption of dry solids in the growth reaction, while the last term on the RHS represents a linear approximation of the water vapor profile within the bioreactor, where $y_{\text {in }}$ and $y_{\text {out }}$ are the inlet and outlet humidities.

The solution of these equations suggests that the axial temperature gradient is highest at the inlet and decreases constantly with axial distance. This, combined with the exponential relationship of water vapor concentration with temperature as described by the Antoine equation, causes the 
water vapor concentration to increase almost linearly with axial distance [16].

\subsection{Key insights obtained from packed-bed bioreactor models and future improvements needed}

The various mathematical models of packed-bed bioreactors described above have been quite useful in investigating possible strategies for large-scale operation. Available operating variables for packed beds are the temperature, flow rate and humidity of the inlet air, and, for bioreactors where wall cooling can make a significant contribution, the position of heat transfer surfaces and the temperature of the cooling water can also be manipulated.

Axial temperature gradients are impossible to avoid within packed-bed bioreactors due to the use of convective cooling with unidirectional flow of air [13]. In fact, these temperature gradients can be considered to be pseudo-steady-state gradients since they depend on the rate of growth of the microorganism and this changes only relatively slowly during the fermentation [16]. The superficial velocity of this air is a key operating variable with higher superficial velocities decreasing the temperature difference between the inlet and outlet [14]. Current models do not recognize an upper limit on the superficial velocities that can be used. Acceptable superficial velocities could depend on considerations of pressure drop across the bed, but experimental studies have not yet been undertaken to define the practical limits in packed-bed bioreactors at large scale.

In wide bioreactors without internal heat transfer plates, the axial temperature gradient imposes a maximum height on the bed if it is desired to maintain the temperature rise over the bioreactor within predefined limits, with this maximum height depending on the maximum growth rate of the microorganism and the superficial air velocity used [14]. Therefore in the scaling up of such packed beds, the bed height can be increased only up to a certain point, after which increases in scale should be achieved with increase in diameter of the bed without further increase in height. Given typical growth kinetics, critical bed heights would be around $1 \mathrm{~m} \mathrm{[14].}$

A model based on Eq. (11) was used to test the effect of periodic reversal of the air flow direction, with the rational being not to let either end of the bed reach high temperatures. However, the model predictions showed that, although this strategy helps to reduce the problem of high temperatures at the bioreactor ends, it causes very high temperatures to occur in the middle regions of the bioreactor, and therefore suggests that such a strategy should not be tried in practice [13].

For bioreactors in which there are internal heat transfer plates, such as the Zymotis bioreactor of Roussos et al. [17], mathematical modeling has been used to show that at large scale relatively small spacings will be required between the heat transfer plates, of the order of $5-10 \mathrm{~cm}$, and that it is essential to control the temperature of the water in the cooling plates [15]. If the cooling water is kept at a constant temperature throughout the fermentation, it is impossible to prevent both overcooling of the bed during the early stages of the fermentation and overheating during the time of peak heat production. The model shows the advantage of the Zymotis bioreactor: the conductive heat removal to the plates minimizes the axial temperature gradient, especially in the upper regions of the bioreactor. The model predictions are consistent with experimental results for a $6 \mathrm{~cm}$ diameter $35 \mathrm{~cm}$ high packed bed with a water jacket, in which there was a significant temperature gradient in the bottom $5 \mathrm{~cm}$ near the air inlet but a relatively small gradient in the upper regions [9].

It is not practical to add water during the fermentation to the static bed within a packed-bed bioreactor. Unfortunately it is impossible to prevent evaporation even if saturated air is used at the air inlet, because the axial temperature gradient increases the water-carrying capacity of the air. In the absence of water addition, it is possible that the substrate can dry sufficiently to cause moisture limitation of growth. Models that include water balances, such as that of Weber et al. [16] are essential to predict the degree of drying that will occur. Although a water balance was not included in the model of the Zymotis bioreactor [15], if it had been it would have shown a further advantage of the Zymotis bioreactor: the smaller axial temperature gradient minimizes the evaporation and therefore the problems of drying out of the substrate bed during the fermentation are decreased.

\section{State of the art in the modeling of intermittently stirred, forcefully aerated bioreactors}

A number of SSF bioreactors have been built that are similar to packed beds in that the substrate bed spends long periods in static mode with forced aeration, the difference being that the bioreactor contains an agitator that allows for intermittent agitation [18-22]. The static periods of operation of such a bioreactor can be expected to have much in common with a packed-bed bioreactor. In fact, Eq. (11) was used to model the energy balance during the static periods in an intermittently stirred forcefully aerated bioreactor [13]. The effect of mixing was modeled by assuming instantaneous mixing events in which the energy content and the biomass content of the bed were averaged over the whole bed. No deleterious effect of mixing on the growth of the microorganism was incorporated into the model.

Simulations with this model suggest that after a mixing event the pseudo-steady-state temperature profile that would be expected during packed-bed operation is reestablished. For a superficial velocity of $0.02 \mathrm{~m} \mathrm{~s}^{-1}$ this would take of the order of $30 \mathrm{~min}$ for a $35 \mathrm{~cm}$ bed [13]. The temperature at the top of the bed can reach values in excess of that expected for packed-bed operation because during the time in which pseudo-steady temperature profile is being reestablished the cooling effect is concentrated in the lower regions of the 
bioreactor. The only way to operate an intermittently agitated bioreactor so as maintain the temperature at the top lower than the pseudo-steady-state temperature is to mix at least every 5 min [13].

Unfortunately, there has been no experimental work done to confirm these predictions. Several improvements to this model are desirable. The effect of mixing on growth should be included but unfortunately the current state of knowledge about the effect of mixing on growth is currently not sufficient to allow the proposal of equations suitable for incorporation into bioreactor models. Also, intermittent mixing events would allow the use of dry air in order to promote evaporation, but in this case the model would need to be rewritten to recognize the substrate and air as separate phases, rather than the current strategy of considering a single pseudo-homogeneous phase with the average properties of the substrate particles and the interparticle air, and to use mass and heat transfer terms to describe heat and mass transfer between these two phases instead of assuming equilibrium.

\section{State of the art in the modeling of rotating-drum bioreactors}

The rotating-drum bioreactor model of Stuart [23] involves three subsystems, the substrate bed, the headspace gases and the bioreactor wall. Each is treated as a well-mixed system. An energy balance is written over each of these (Fig. 4). The energy balance over the substrate bed is

$$
\begin{aligned}
& \frac{\mathrm{d}\left[T_{\mathrm{s}} M\left(C_{p \mathrm{~m}}+C_{p \mathrm{w}} W\right)\right]}{\mathrm{d} t} \\
& \quad=r_{Q}-h_{\mathrm{sf}} A_{\mathrm{sf}}\left(T_{\mathrm{s}}-T_{\mathrm{f}}\right)-h_{\mathrm{sa}} A_{\mathrm{sa}}\left(T_{\mathrm{s}}-T_{\mathrm{a}}\right) \\
& \quad-k A_{\mathrm{sa}}\left(C_{\mathrm{I}}-C_{\mathrm{B}}\right)\left(T_{\mathrm{s}} C_{p \mathrm{w}}+\lambda-\left(T_{\mathrm{s}}-T_{\mathrm{a}}\right) C_{p \mathrm{VAP}}\right)
\end{aligned}
$$

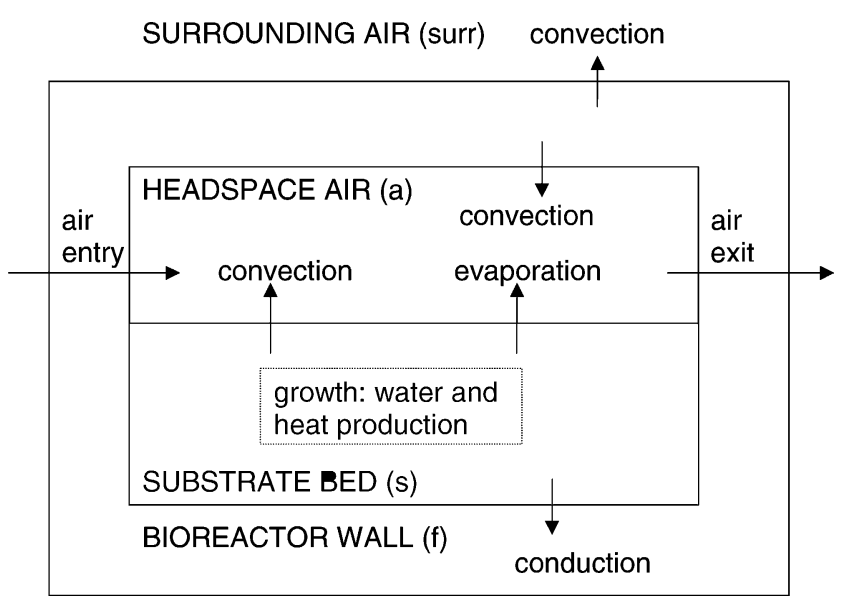

Fig. 4. The various subsystems in a rotating-drum bioreactor (with the subscripts used by Stuart [23]) and the mass and heat exchanges between them. where on the LHS of the equation $T_{\mathrm{S}}$ is the temperature of the substrate bed and $M$ is its dry mass, $C_{p m}$ the heat capacity of the dry solids, $W$ the water content of the bed and $C_{p \mathrm{w}}$ the heat capacity of liquid water. The second term on the RHS describes heat transfer from the substrate bed to the bioreactor wall, where $h_{\mathrm{sf}}$ is the heat transfer coefficient, $A_{\text {sf }}$ the area of contact and $T_{\mathrm{f}}$ the wall temperature. The third term describes convective heat transfer from the substrate bed to the headspace air, where $h_{\mathrm{sa}}$ is the heat transfer coefficient, $A_{\mathrm{sa}}$ the area of contact and $T_{\mathrm{a}}$ the headspace air temperature. The fourth term describes evaporative heat removal where $k$ is the mass transfer coefficient, $C_{\mathrm{I}}$ the vapor concentration in equilibrium with the solids, $C_{\mathrm{B}}$ the vapor concentration of the headspace. The symbols $\lambda$ and $C_{p \mathrm{VAP}}$ have been previously defined.

The energy balance over the headspace is

$$
\begin{aligned}
\frac{\mathrm{d}\left[T_{\mathrm{a}}\right.}{\left.G\left(C_{p \mathrm{~g}}+C_{p \mathrm{VAP}} H\right)\right]} & \mathrm{d} t \\
= & T_{\mathrm{i}} F_{\mathrm{i}}\left(C_{p \mathrm{~g}}+C_{p \mathrm{VAP}} H_{\mathrm{i}}\right)-T_{\mathrm{a}} F_{\mathrm{o}}\left(C_{p \mathrm{~g}}+C_{p \mathrm{VAP}} H\right) \\
& +k A_{\mathrm{sa}}\left(C_{\mathrm{I}}-C_{\mathrm{B}}\right) T_{\mathrm{a}} C_{p \mathrm{VAP}}+h_{\mathrm{sa}} A_{\mathrm{sa}}\left(T_{\mathrm{s}}-T_{\mathrm{a}}\right) \\
& +h_{\mathrm{fa}} A_{\mathrm{fa}}\left(T_{\mathrm{f}}-T_{\mathrm{a}}\right)
\end{aligned}
$$

where on the LHS of the equation $G$ is the mass of dry gas in the headspace and $H$ the humidity in the headspace. The first two terms on the RHS are the sensible energy entering and leaving the bioreactor in the air stream, where $T_{\mathrm{i}}, F_{\mathrm{i}}$ and $H_{\mathrm{i}}$ are the temperature, flow rate and humidity of the inlet air, $C_{p \mathrm{~g}}$ the heat capacity of dry air and $F_{\mathrm{o}}$ the outlet air flow rate. The third term takes into account sensible energy changes of the water that evaporates into the headspace. The fourth term describes convective heat transfer from the bed to the headspace, being identical to the term in Eq. (15) but with opposite sign. The last term describes the convective heat transfer between the bioreactor wall and the headspace air, where $h_{\mathrm{fa}}$ is the heat transfer coefficient and $A_{\mathrm{fa}}$ the area of contact between the wall and the headspace air.

The energy balance over the bioreactor wall gives

$$
\begin{aligned}
\frac{\mathrm{d}\left(T_{\mathrm{f}} V_{\mathrm{f}} \rho_{\mathrm{f}} C_{\mathrm{pf}}\right)}{\mathrm{d} t}= & h_{\mathrm{sf}} A_{\mathrm{sf}}\left(T_{\mathrm{s}}-T_{\mathrm{f}}\right)-h_{\mathrm{fa}} A_{\mathrm{fa}}\left(T_{\mathrm{f}}-T_{\mathrm{a}}\right) \\
& -h_{\mathrm{fe}} A_{\mathrm{fe}}\left(T_{\mathrm{f}}-T_{\mathrm{e}}\right)
\end{aligned}
$$

where on the LHS $V_{\mathrm{f}}$ is the volume of metal in the body of the bioreactor, $\rho_{\mathrm{f}}$ its density and $C_{p \mathrm{f}}$ its heat capacity. The first term on the RHS describes heat transfer between the substrate bed and the bioreactor wall and the second term describes heat transfer between the headspace and the bioreactor wall. These terms appeared in Eqs. (15) and (16) with opposite signs. The third term describes heat transfer between the bioreactor wall and the external air, where $h_{\mathrm{fe}}$ is the heat transfer coefficient, $A_{\mathrm{fe}}$ the area of contact between the bioreactor wall and the external air and $T_{\mathrm{e}}$ the temperature of the external air.

The mass balance for water over the substrate bed is

$\frac{\mathrm{d} M W}{\mathrm{~d} t}=-k A_{\mathrm{sa}}\left(C_{\mathrm{I}}-C_{\mathrm{B}}\right)+r_{\mathrm{H}_{2} \mathrm{O}}$ 
where the first term on the RHS describes the loss of water from the substrate bed by evaporation and the second term the production of metabolic water as a result of growth. Another mass balance can be written for water over the headspace:

$\frac{\mathrm{d} G H}{\mathrm{~d} t}=F_{\mathrm{i}} H_{\mathrm{i}}-F_{\mathrm{o}} H+k_{\mathrm{w}} A_{\mathrm{sa}}\left(C_{\mathrm{i}}-C_{\mathrm{b}}\right)$

where the three terms on the RHS represent the entry of water with the inlet air, the exit of water with the outlet air and evaporation from the bed into the headspace air.

The model predictions of temperature profiles for the substrate, bioreactor wall and headspace gas agreed reasonably well with experimental results for SSFs in a laboratory scale rotating-drum bioreactor [23]. The fact that the model recognized the bioreactor wall as a separate system, something which is rarely done in modeling of SSF bioreactors, allowed it to identify that the wall temperature will be higher than the headspace temperature, such that the direction of transfer is from the wall not only to the outside air, but also to the headspace gases. This heating of the headspace air reduces its convective cooling capacity but increases its evaporative cooling capacity.

Simulations with the model suggest that heat removal through the bioreactor wall to the surrounding air can be important at small scale, but that at large-scale evaporation must be promoted in order to provide rapid cooling. For example, the model suggests that a rotating-drum bioreactor of $20 \mathrm{~m}^{3}$ containing $2000 \mathrm{~kg}$ of fermenting substrate would require aeration rates of the order of eight volumes of air per bioreactor volume per minute, with the air being almost dry, in order to control the temperature of the bed adequately.

Several improvements could be made to this model. The model assumes that the bed volume remains constant during the fermentation, despite the loss of substrate dry matter as $\mathrm{CO}_{2}$. Volume losses could be modeled if it were known how the particle size reduced during the fermentation [2]. More importantly, the effective area for transport between the substrate bed and headspace air and how this depends on rotation rate is not known. Stuart [23] simply assumed a fivefold increase in transfer efficiency, but without any experimental data. Experimental work is needed in order to develop correlations of transfer coefficients with rotational speeds and with the presence and absence of lifters, which can then be incorporated into models of rotating-drum bioreactors.

It will not always necessarily be the case that rotatingdrum bioreactors can be considered to be well mixed, especially when large length to diameters are used and no special efforts are made to promote axial mixing. In such cases, axial dispersion of particles can be reasonably slow [24]. It is a relatively simple matter to assume perfect mixing in the radial direction but no or limited mixing in the axial direction, producing a model based on partial differential equations, although this has not yet been done. Likewise, the gas headspace will typically not be perfectly mixed and experimental work needs to be undertaken to characterize gas flow patterns in the headspace, as has been done by Hardin et al. [25].

\section{State of the art of modeling well-mixed bioreactors with forced aeration}

The difference between the bioreactors discussed in this section and rotating-drum bioreactors is in the manner in which the bed is aerated. In forcefully aerated bioreactors the gas is blown directly into the substrate bed, improving the contact between the gas and the substrate particles, whereas in the rotating-drum the bed/headspace surface can limit transport. In the case of gas-solid fluidized bed bioreactors, typically the bioreactor can not only be assumed to be well mixed, but also convective heat removal is typically so effective that the energy balance is not even considered. For this reason, the models of these bioreactors focus on the intraparticle processes [26,27], which are discussed elsewhere [2]. The first model proposing water and energy balances for a well mixed forcefully aerated bioreactor was that of Sargantanis et al. [28]. More recent developments are described below.

\subsection{A model used as a softsensor for on-line estimation of water content}

Pena y Lillo et al. [29] used a model that assumed perfect mixing in order to use indirect on-line measurements to estimate the water content of the substrate bed. This allows water content to be used as the control variable even though on-line measurement of water content itself is not possible.

The overall energy balance is

$$
\begin{aligned}
C_{p \text { tot }} & k_{\exp } \frac{\mathrm{d} T}{\mathrm{~d} t} \\
= & r_{Q}-\left[F _ { \text { air } } \left(C_{p \mathrm{~g}}\left(T_{\mathrm{o}}-T_{\mathrm{i}}\right)+C_{p \operatorname{VAP}}\left(y_{\text {out }} T_{\mathrm{o}}-y_{\text {in }} T_{\mathrm{i}}\right)\right.\right. \\
& \left.-C_{p \operatorname{VAP}} T\left(y_{\text {out }}-y_{\text {in }}\right)\right]-F_{\text {air }} \lambda\left(y_{\text {out }}-y_{\text {in }}\right) \\
& -\left[h A\left(T-T_{\text {surr }}\right)+h \sigma \epsilon\left(T^{4}-T_{\text {surr }}^{4}\right)\right] \\
& +F_{\mathrm{w}} C_{p \mathrm{w}}\left(T_{\mathrm{w}}-T\right)
\end{aligned}
$$

On the LHS, $C_{p \text { tot }}$ is the total heat capacity of the bioreactor, including not only the moist solid bed but also the metal of the bioreactor walls. The contribution of the bioreactor wall to heat accumulation is typically neglected in bioreactor models [1], but can be significant in large-scale bioreactors [29]. The parameter $k_{\exp }$ is an empirical parameter that was necessary to account for errors introduced by the assumption of bed homogeneity, which was not achieved in practice. The second term on the RHS, that is, within the first set of square brackets, represents convective heat removal, where all the symbols are used as defined for previous models. The third term on the RHS represents evaporation. The term in the second set of square brackets represents heat transfer to the surroundings by natural convection and radiation, with the wall 
temperature being assumed equal to the bed temperature, where $h$ is the convective heat transfer coefficient, $A$ the wall area, $T_{\text {surr }}$ the temperature of the surroundings, $\epsilon$ the emissivity of the bioreactor walls and $\sigma$ the Stefan-Boltzmann constant. The last term represents enthalpy changes associated with the seepage of water from the bed at the temperature of the bed and addition of water at temperature $T_{\mathrm{w}}$.

The balance for the water content of the substrate bed, $X_{\mathrm{w}}$, in kilograms of water per kilograms of dry solids, is expressed as

$\frac{\mathrm{d} X_{\mathrm{w}}}{\mathrm{d} t}=r_{\mathrm{H}_{2} \mathrm{O}}+\frac{F_{\mathrm{w}}}{M_{\mathrm{s}}}-\frac{W_{\mathrm{p}}}{M_{\mathrm{S}}}-\frac{F_{\text {air }}\left(y_{\text {out }}-y_{\text {in }}\right)}{M_{\mathrm{S}}}-\frac{X_{\mathrm{w}}}{M_{\mathrm{S}}} \frac{\mathrm{d} M_{\mathrm{s}}}{\mathrm{d} t}$

where $r_{\mathrm{H}_{2} \mathrm{O}}$ represents the rate of metabolic production of water, which can be estimated from the $\mathrm{CO}_{2}$ evolution rate with the known stoichiometry for the growth reaction. The second term on the RHS represents the addition of water, the third term the rate of water seepage from the bed, and the fourth term the rate of evaporation. $M_{\mathrm{S}}$ is the total mass of dry solids. The last term accounts for the effect on the water content of the loss of dry weight of these solids during the growth process in the form of $\mathrm{CO}_{2}$.

With experimental measurements of the $\mathrm{CO}_{2}$ production rate and the conditions of the inlet and outlet air, it was possible to use this model to predict the bed temperature and water content. The bed temperature was measured on-line and compared with the measured values. In experiments conducted with a $200 \mathrm{~kg}$ capacity pilot scale bioreactor significant deviations occurred between the measured and predicted temperatures, these deviations being attributed to measurement errors. On the other hand, predicted bed water contents agreed closely with values measured off-line, indicating that the model can be a useful softsensor for use in control of bioreactor water content [29].

\subsection{An extracellular water balance model for use in controlling water content}

Nagel et al. [30], recognizing the fact that the biomass water content and the water content of the residual substrate can be quite different, proposed a model in which the water balance was used to estimate the extracellular water content, which was used as a control variable in their control scheme. Extracellular, intracellular and headspace water pools were recognized (Fig. 5).

The balance describing the change in the mass of extracellular water within the bioreactor $\left(W_{\mathrm{ex}}\right)$ is

$$
\begin{aligned}
\frac{\mathrm{d} W_{\mathrm{ex}}}{\mathrm{d} t}= & F_{\mathrm{air}}\left(C_{\mathrm{w} \text { in }}-C_{\mathrm{w} \text { out }}\right)-X_{\mathrm{wx}} Y_{\mathrm{x} / \mathrm{O}_{2}} r_{\mathrm{O}_{2}} \mathrm{MW}_{\mathrm{x}} \\
& +Y_{\mathrm{w} / \mathrm{O}_{2}} r_{\mathrm{O}_{2}} \mathrm{MW}_{\mathrm{w}}-Y_{\mathrm{hyd}} Y_{\mathrm{S} / \mathrm{O}_{2}} r_{\mathrm{O}_{2}}
\end{aligned}
$$

The first term on the RHS represents the entry and exit of water in the inlet and exit air streams, where $C_{\mathrm{w}}$ in and $C_{\mathrm{w} \text { out }}$ are the humidities of these streams. The second term

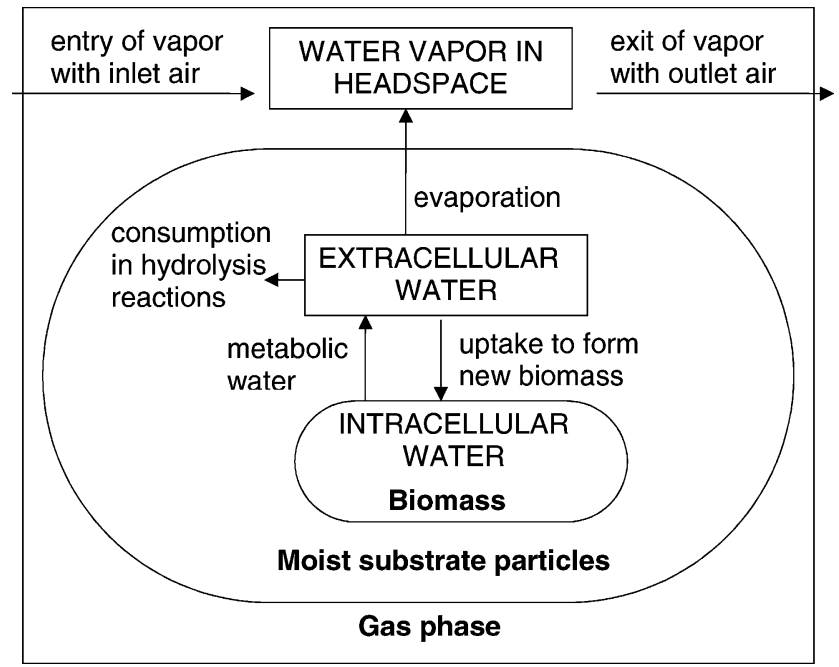

Fig. 5. The various pools of water and the transfers between them, as modeled by Nagel et al. [31].

represents the uptake of water into the biomass, where $X_{\mathrm{wx}}$ is the water content of the biomass, $Y_{\mathrm{x} / \mathrm{O}_{2}}$ is the yield of biomass from $\mathrm{O}_{2}$ and $\mathrm{MW}_{\mathrm{x}}$ is the molecular weight of the biomass. The third term represents water production as a result of respiration by the microorganism, where $Y_{\mathrm{w} / \mathrm{O}_{2}}$ is the yield of water from $\mathrm{O}_{2}$ and $\mathrm{MW}_{\mathrm{w}}$ the molecular weight of water. The fourth term represents the utilization of water in the hydrolysis of starch, where $Y_{\text {hyd }}$ is the water required to hydrolyze the starch and $Y_{\mathrm{S} / \mathrm{O}_{2}}$ gives the relationship between starch hydrolysis and $\mathrm{O}_{2}$ consumption.

Since it is impossible to measure the extracellular water experimentally, it is necessary to use the model to predict the overall moisture content of the solid bed, allowing the model predictions to be compared against a variable that can be measured. To do this, it is necessary to describe the change in the dry weight of the residual wheat substrate $\left(M_{\mathrm{wh}}\right)$ as a function of time:

$\frac{\mathrm{d} M_{\mathrm{wh}}}{\mathrm{d} t}=-\left(\frac{Y_{\mathrm{S} / \mathrm{O}_{2}}}{Y_{\mathrm{CO}_{2} / \mathrm{O}_{2}}} \mathrm{MW}_{\mathrm{S}}+\frac{Y_{\mathrm{N} / \mathrm{O}_{2}}}{Y_{\mathrm{CO}_{2} / \mathrm{O}_{2}}} \mathrm{MW}_{\mathrm{N}}\right) r_{\mathrm{CO}_{2}}$

The first term in the parenthesis represents the loss of matter from starch as $\mathrm{CO}_{2}$ while the second term represents the loss of matter from protein as $\mathrm{CO}_{2} . \mathrm{MW}_{\mathrm{S}}$ and $\mathrm{MW}_{\mathrm{N}}$ are the molecular weights of the starch and protein in the substrate, respectively, per carbon mole. The yield coefficients are self-explanatory.

The overall moisture content of the solid bed $\left(X_{\mathrm{w} \text { overall }}\right)$ is then given as

$X_{\mathrm{w} \text { overall }}=\frac{W_{\mathrm{wh}}+W_{\mathrm{x}}}{M_{\mathrm{wh}}+M_{\mathrm{x}}}$

where $M_{\mathrm{x}}$ is the total mass of dry biomass, given by the measured growth kinetics, and $W_{\mathrm{x}}$ the total amount of intracellular water, calculated on the basis of a constant biomass water content. 
In this work, the rate of $\mathrm{CO}_{2}$ production was measured experimentally, however, it could also be related to a growth kinetic equation. The model simulated well the changes in the overall water content for two different fermentations in a well-stirred scraped-drum bioreactor: one fermentation in which evaporative cooling was promoted and another fermentation in which evaporative cooling was minimized by maximizing the removal of heat through the bioreactor wall. In using this model within a bioreactor control scheme, control aimed at constant extracellular water content was found to be superior to control aimed at constant overall water content of the fermented solids [31].

\section{Use of simplified models for guiding the scale up of bioreactors}

Saucedo-Castaneda et al. [32] pointed out that if the energy and moisture balance equations were equated to zero and if this equality could be maintained with scale then the balance equation could be used to guide the construction of large-scale bioreactors. For packed-bed bioreactors, this approach has been applied to the energy balance with the proposal of a modified Damkohler number [14], and to both the water and energy balances [16]. It has also been used to show that at large-scale well-mixed bioreactors should perform better than packed-bed bioreactors [33] and to investigate the scale up of rotating-drum bioreactors [34]. However, despite the fact that such approaches might be attractive from the sake of simplicity, full dynamic models will always be much more powerful tools for guiding the optimization of bioreactor performance.

\section{Conclusion}

Over the past decade or so, there have been significant advances in the modeling of heat and mass transfer phenomena in SSF bioreactors, with models having been developed for all the major bioreactor types. Further, although initial work tended to focus on the energy balance, with the aim of preventing high temperatures, current models consider both the energy balance and the water balance, allowing their use in bioreactor control schemes designed to maintain both bed temperature and water content at the optimum values for growth and product formation. Various improvements that are desirable for the current models of specific bioreactor types have already been identified above. Some of the future advances in modeling of bioreactors may come from describing the kinetics of growth and the phenomena at the particle level in a less empirical manner than is done presently. This issue is discussed in Part II of this review [2].

Currently there are no reports in the literature describing the use of mathematical models in the construction of large-scale bioreactors. However, mathematical models have now reached a level of sophistication which makes this not only possible, but also necessary; it is only through the use of mathematical models as tools during the design process and in the optimization of operation that SSF bioreactors will perform at their full potential and thereby maximize the economic performance of SSF processes.

\section{Acknowledgements}

The present work was done with the financial support of CNPq (Conselho Nacional de Desenvolvimento Científico e Tecnológico), a unit of the Brazilian government responsible for scientific and technological development. David Mitchell also thanks CNPq for a research scholarship.

\section{References}

[1] D.A. Mitchell, D.M. Stuart, R.D. Tanner, Solid-state fermentationmicrobial growth kinetics, in: M.C. Flickinger, S.W. Drew (Eds.), The Encyclopedia of Bioprocess Technology: Fermentation, Biocatalysis and Bioseparation, vol. 5, Wiley, New York, 1999, pp. 24072429.

[2] D.A. Mitchell, O.F. von Meien, N. Krieger, Recent advances in modeling of solid-state fermentation. II. Modeling of microbial growth kinetics, Biochem. Eng. J., submitted for publication.

[3] K.S.M.S. Ragheva Rao, M.K. Gowthaman, N.P. Ghildyal, N.G. Karanth, A mathematical model for solid state fermentation in tray bioreactors, Bioprocess Eng. 8 (1993) 255-262.

[4] K.W. Szewczyk, The influence of heat and mass transfer on solid state fermentation, Acta Biochim. Polon. 40 (1993) 90-92.

[5] S. Rajagopalan, J.M. Modak, Heat and mass transfer simulation studies for solid-state fermentation processes, Chem. Eng. Sci. 49 (1994) 2187-2193.

[6] S. Rajagopalan, J.M. Modak, Modeling of heat and mass transfer for solid state fermentation process in tray bioreactor, Bioprocess Eng. 13 (1995) 161-169.

[7] J.P. Smits, H.M. van Sonsbeek, J. Tramper, W. Knol, W. Geelhoed, M. Peeters, A. Rinzema, Modelling fungal solid-state fermentation: the role of inactivation kinetics, Bioprocess Eng. 20 (1999) 391404.

[8] D.A. Mitchell, M. Berovic, N. Krieger, Biochemical engineering aspects of solid state bioprocessing, Adv. Biochem. Eng. 68 (2000) 61-138.

[9] G. Saucedo-Casteneda, M. Gutierrez-Rojas, G. Bacquet, M. Raimbault, G. Viniegra-Gonzalez, Heat transfer simulation in solid substrate fermentation, Biotechnol. Bioeng. 35 (1990) 802-808.

[10] P. Sangsurasak, D.A. Mitchell, Incorporation of death kinetics into a 2-D dynamic heat transfer model for solid state fermentation, J. Chem. Technol. Biotechnol. 64 (1995) 253-260.

[11] S.D.M. Hasan, J.A.V. Costa, A.V.L. Sanzo, Heat transfer simulation of solid state fermentation in a packed-bed bioreactor, Biotechnol. Tech. 12 (1998) 787-791.

[12] P. Sangsurasak, D.A. Mitchell, Validation of a model describing 2-dimensional heat transfer during solid-state fermentation in packed bed bioreactors, Biotechnol. Bioeng. 60 (1998) 739-749.

[13] V.M. Ashley, D.A. Mitchell, T. Howes, Evaluating strategies for overcoming overheating problems during solid state fermentation in packed bed bioreactors, Biochem. Eng. J. 3 (1999) 141-150.

[14] D.A. Mitchell, A. Pandey, P. Sangsurasak, N. Krieger, Scale-up strategies for packed-bed bioreactors for solid-state fermentation, Process Biochem. 35 (1999) 167-178. 
[15] D.A. Mitchell, O.F. von Meien, Mathematical modeling as a tool to investigate the design and operation of the Zymotis packed-bed bioreactor for solid-state fermentation, Biotechnol. Bioeng. 68 (2000) $127-135$.

[16] F.J. Weber, J. Tramper, A. Rinzema, A simplified material and energy balance approach for process development and scale-up of Coniothyrium minitans conidia production by solid-state cultivation in a packed-bed reactor, Biotechnol. Bioeng. 65 (1999) 447-458

[17] S. Roussos, M. Raimbault, J.-P. Prebois, B.K. Lonsane, Zymotis, a large scale solid state fermenter, Appl. Biochem. Biotechnol. 42 (1993) 37-52.

[18] A. Durand, D. Chereau, A new pilot reactor for solid-state fermentation: application to the protein enrichment of sugar beet pulp, Biotechnol. Bioeng. 31 (1988) 476-486.

[19] M. Xue, D. Liu, H. Zhang, Q. Hongyan, Z. Lei, A pilot process of solid state fermentation from sugar beet pulp for the production of microbial protein, J. Ferment. Bioeng. 73 (1992) 203-205.

[20] Y. Chamielec, R. Renaud, J. Maratray, S. Almanza, M. Diezand, A. Durand, Pilot-scale reactor for aseptic solid-state cultivation, Biotechnol. Tech. 8 (1994) 245-248.

[21] E. Agosin, R. Perez-Correa, M. Fernandez, I. Solar, L. Chiang, in: D.L. Wise (Ed.), An Aseptic Pilot Bioreactor for Solid Substrate Cultivation Processes, Kluwer Academic Publishers, Dordrecht, 1997, pp. 233-243.

[22] S. Bandelier, R. Renaud, A. Durand, Production of gibberellic acid by fed-batch solid state fermentation in an aseptic pilot-scale reactor, Process Biochem. 32 (1997) 141-145.

[23] D.M. Stuart, Solid-state fermentation in rotating drum bioreactors, Ph.D. Thesis, The University of Queensland, Brisbane, Australia 1996.

[24] A.J. Marsh, D.M. Stuart, D.A. Mitchell, T. Howes, Characterizing mixing in a rotating drum bioreactor for solid-state fermentation, Biotechnol. Lett. 22 (2000) 473-477.
[25] M.T. Hardin, D.A. Mitchell, T. Howes, Residence time distributions of gas flowing through rotating drum bioreactors, Biotechnol. Bioeng. 74 (2001) 145-153

[26] M. Menner, D. Bahr, Solid-state-fermentation of starter cultures in fluidized bed. Part 2. Mathematical modeling, Bioforum 18 (1995) 366-372.

[27] L. Rottenbacher, M. Schossler, W. Bauer, Modelling a solid-state fluidized bed fermenter for ethanol production with Saccharomyces cerevisiae, Bioprocess Eng. 2 (1987) 25-31.

[28] J. Sargantanis, M.N. Karim, V.G. Murphy, D. Ryoo, R.P. Tengerdy, Effect of operating conditions on solid substrate fermentation, Biotechnol. Bioeng. 42 (1993) 149-158.

[29] M. Pena y Lillo, R. Perez-Correa, E. Agosin, E. Latrille, Indirect measurement of water content in an aseptic solid substrate cultivation pilot-scale bioreactor, Biotechnol. Bioeng. 76 (2001) 44-51.

[30] F.J.I. Nagel, J. Tramper, S.N. Marjolein, M.S.N. Bakker, A. Rinzema, Model for on-line moisture-content control during solid-state fermentation, Biotechnol. Bioeng. 72 (2001) 231-243.

[31] F.J.I. Nagel, J. Tramper, M.S.N. Bakker, A. Rinzema, Simultaneous control of temperature and moisture content in a mist bioreactor for solid-state fermentation, Biotechnol. Bioeng., in press.

[32] G. Saucedo-Castaneda, B.K. Lonsane, M.M. Krishnaiah, J.M. Navarro, S. Roussos, M. Raimbault, Maintenance of heat and water balances as a scale-up criterion for the production of ethanol by Schwanniomyces castelli in a solid state fermentation system, Process Biochem. 27 (1992) 97-107.

[33] J. Oostra, J. Tramper, A. Rinzema, Model-based bioreactor selection for large-scale solid-state cultivation of Coniothyrium minitans spores on oats, Enzyme Microb. Technol. 27 (2000) 652-663.

[34] M.T. Hardin, D.A. Mitchell, T. Howes, An approach to designing rotating drum bioreactors for solid-state fermentation on the basis of dimensionless design factors, Biotechnol. Bioeng. 67 (2000) 274 282 